Research Article

\title{
Recanalization Treatment of Acute Ischemic Stroke Caused by Large-Artery Occlusion in the Elderly: A Comparative Analysis of "the Elderly" and "the Very Elderly"
}

\author{
Qi Wang, ${ }^{1,2}$ Yi-Qun Zhang, ${ }^{3}$ Han-Cheng Qiu, ${ }^{3}$ Yin-Dan Yao, ${ }^{3}$ Ao-Fei Liu, ${ }^{3}$ Chen Li, ${ }^{3}$ \\ and Wei-Jian Jiang $\mathbb{B}^{1,3}$ \\ ${ }^{1}$ The PLA Rocket Force Characteristic Medical Center, The Teaching Hospital of Soochow University, Beijing, China \\ ${ }^{2}$ Department of Vascular Neurosurgery, New Era Stroke Care and Research Institute, Department of Neurology, \\ Beijing Rehabilitation Hospital, Capital Medical University, Beijing, China \\ ${ }^{3}$ Department of Vascular Neurosurgery, New Era Stroke Care and Research Institute, The PLA Rocket Force Characteristic \\ Medical Center, Beijing, China
}

Correspondence should be addressed to Wei-Jian Jiang; jiangweijian2018@163.com

Received 11 June 2021; Revised 27 August 2021; Accepted 31 August 2021; Published 6 October 2021

Academic Editor: Xianwei Zeng

Copyright (c) 2021 Qi Wang et al. This is an open access article distributed under the Creative Commons Attribution License, which permits unrestricted use, distribution, and reproduction in any medium, provided the original work is properly cited.

Objective. To assess whether the effectiveness and safety of recanalization therapy for acute ischemic stroke (AIS) caused by largeartery occlusion (LAO) differ between patients aged 60-79 years and patients aged $\geq 80$ years. Methods. We analyzed prospective data of patients with LAO ( $\geq 60$ years) who underwent recanalization therapy at the Department of Vascular Neurosurgery, New Era Stroke Care and Research Institute, PLA Rocket Force Characteristic Medical Center, from November 2013 to July 2017. The data were compared between elderly patients (60-79 years) and very elderly patients ( $\geq 80$ years). The effectiveness of recanalization therapy was evaluated using the 90-day modified Rankin scale (mRS) score, while safety was assessed by the rates of symptomatic intracranial hemorrhage (SICH) and mortality within 30 days. Results. A total of 151 patients with AIS induced by LAO were included in this study. Seventy-three patients $(48.3 \%$ [73/151]) had an overall favorable outcome (mRS score $0-2$ ) after treatment. A higher proportion of patients in the elderly group showed a favorable outcome compared with the very elderly group (58.6\% [34/58] vs. $41.6 \%$ [39/93], respectively; $P=0.046)$. The incidence of SICH $(12.7 \%$ vs. $16.13 \%$, respectively; $P=0.561)$ and mortality $(10.3 \%$ vs. $7.5 \%$, respectively; $P=0.548)$ within 30 days was not significantly different between the two groups. Conclusion. Recanalization treatment of LAO is more effective in elderly patients compared with very elderly patients, while the safety of recanalization treatment is comparable between these two groups.

\section{Introduction}

Acute large-artery occlusion (LAO) has become the most important cause of acute ischemic stroke (AIS) worldwide and is related to the high recurrence rate of ischemic stroke and poor outcomes [1]. Endovascular thrombectomy (ET) and intravenous alteplase thrombolysis (IVT) are currently the preferred vascular recanalization treatments for AIS.

Stroke morbidity and mortality increase with age, and the absolute number of patients with fatal stroke is likely to increase steadily due to population aging, a continued increase in traditional risk factors, and poor management [2].
Age is usually regarded as an important factor affecting the choice of treatment and prognosis, and this consideration is particularly prominent in individuals aged over 80 years [3-6]. However, few randomized controlled trials have investigated large-vessel recanalization treatment in patients aged over 80 years.

With the development of recanalization treatment technology and population aging, the treatment of acute LAO in patients over 80 years of age is receiving increasing attention. In 2013, the Chinese Stroke Association and the American Heart Association/American Stroke Association modified AIS recombinant tissue plasminogen activator (rt-PA) IVT 
guidelines. Specifically, an age of greater than 80 years was no longer an absolute exclusion criterion; instead, it became a relative exclusion criterion (level II evidence and grade $\mathrm{B}$ recommendation) [7,8]. Moreover, the inclusion criteria for AIS recanalization therapy in clinical practice have been further expanded. Very elderly patients with low National Institutes of Health Stroke Scale (NIHSS) scores, an Alberta Stroke Program Early Computed Tomography (ASPECT) score of $<6$, and posterior circulation infarction have been gradually indicated to undergo revascularization therapy.

At the same time, an increasing number of single-center trials began to include very elderly patients over 80 years of age into the scope of intravascular recanalization treatment and compared them with elderly patients aged less than 80 years [9]. It has been emphasized that the safety of ET is comparable between patients aged over 80 years and those aged less than 80 years [10]. However, the relationship between recanalization therapy and clinical outcomes in very elderly patients (those aged over 80 years of age) is not fully understood. Therefore, this study was aimed at assessing whether the benefits and safety of recanalization therapy for acute LAO and AIS are comparable between patients $\geq 80$ years of age (very elderly patients) and patients aged 60-79 years (elderly patients) to provide a basis for clinical treatment. Thus, we aimed to assess whether there is a difference in the effectiveness and safety of recanalization therapy for AIS caused by LAO between elderly patients and very elderly patients. We also aimed to provide a basis for the future selection of patients suitable for acute stroke recanalization treatment and to investigate whether recanalization treatment has a better effect in elderly patients compared with very elderly patients. The findings of this study may inform future treatment selection.

\section{Materials and Methods}

2.1. Patient Information. This study was a retrospective study that included patients aged $\geq 60$ years with AIS who were admitted continuously to the Department of Vascular Neurosurgery, New Era Stroke Care and Research Institute, PLA Rocket Force Characteristic Medical Center, from November 2013 to July 2017. Patients were divided into the elderly group (60-79 years) and the very elderly group ( $\geq 80$ years). Patients' baseline characteristics, imaging results, and treatment plans were recorded. This study was approved by the Ethics Committee of PLA Rocket Force Characteristic Medical Center, and written informed consent was obtained from all patients.

The inclusion criteria were as follows: (1) aged $\geq 60$ years; (2) met the AIS diagnostic criteria, including clear neurological deficit, presence of an infarct on diffusionweighted imaging, and no cerebral hemorrhage on head computed tomography (CT) [11]; (3) presence of LAO at the internal carotid artery (ICA), vertebral artery, basilar artery (BA), or main middle cerebral artery (M1), which was related to the stroke event and confirmed by magnetic resonance angiography or digital subtraction angiography; and (4) an onset time window of within 4.5 hours for IVT and within 6 hours for intravascular treatment in patients with anterior-circulation disease and within 24 hours for patients with posterior-circulation disease.

The exclusion criteria were as follows: (1) coagulation dysfunction, mental disorders, or malignant tumors; (2) liver or kidney dysfunction, organ diseases, or an incomplete medical history; (3) acute cerebral hemorrhage, systemic infection, or blood system disease; (4) bilateral or multiple large-artery disease, perforating artery disease or small-artery disease, a history of surgical or interventional treatment for aortic valve stenosis, or other diseases affecting clinical follow-up and judgment; (5) refusal or failure to comply with the treatment plan or failure to complete follow-up; and (6) participation in other clinical trials.

\subsection{Treatment Plan}

2.2.1. IVT. For patients who met the requirements for IVT and who had no contraindications to IVT, $0.9 \mathrm{mg} / \mathrm{kg} \mathrm{rt}-\mathrm{PA}$ (Boehringer Ingelheim Pharmaceutical Co., Ltd., Germany) was administered. For patients with a higher bleeding risk, the doctor exercised the decision to administer $0.6 \mathrm{mg} / \mathrm{kg}$ rt-PA based on experience.

2.2.2. ET. All surgeries were performed by an experienced neurointerventionist (Professor Jiang Weijian). General or local anesthesia was used depending on the patient's condition and tolerance. Thrombus location was determined using angiography, and the Solitaire $\mathrm{AB}$ stent removal device was preferentially used for thrombus removal. The degree of recanalization was measured by modified thrombolysis in cerebral infarction (mTICI), with grade $\geq 2 b$ representing successful recanalization and grade 3 representing complete recanalization [12].

2.2.3. Blood Pressure Management. To prevent ischemiareperfusion injury after recanalization, blood pressure was kept below 180/105 $\mathrm{mmHg}$ before surgery and maintained at around $80 \%$ of the baseline level but not less than 90/60 $\mathrm{mmHg}$ after vascular recanalization.

2.2.4. Antiplatelet Drugs. After ET, patients were routinely treated with dual antiplatelet therapy (oral aspirin $100 \mathrm{mg} /$ day and clopidogrel $75 \mathrm{mg}$ /day). Three months later, single-agent antiplatelet therapy with aspirin $100 \mathrm{mg}$ /day or clopidogrel $75 \mathrm{mg}$ /day was administered long term.

2.2.5. Statins. Patients diagnosed with cerebral infarction were immediately administered statin treatment. For patients with severe atherosclerosis or emergency stent implantation, intensive statin treatment was used.

2.3. Outcome Assessment. The mRS score 90 days after treatment was used as the evaluation standard to determine recovery of neurological function. An mRS score of 0-2 was defined as a good functional prognosis, and an mRS score of 3-6 was defined as a poor prognosis [13]. The mRS score was recorded by a neurologist who followed up patients by telephone or during clinic visits.

The incidence of SICH and mortality within 30 days after treatment was recorded. SICH was defined as subarachnoid 


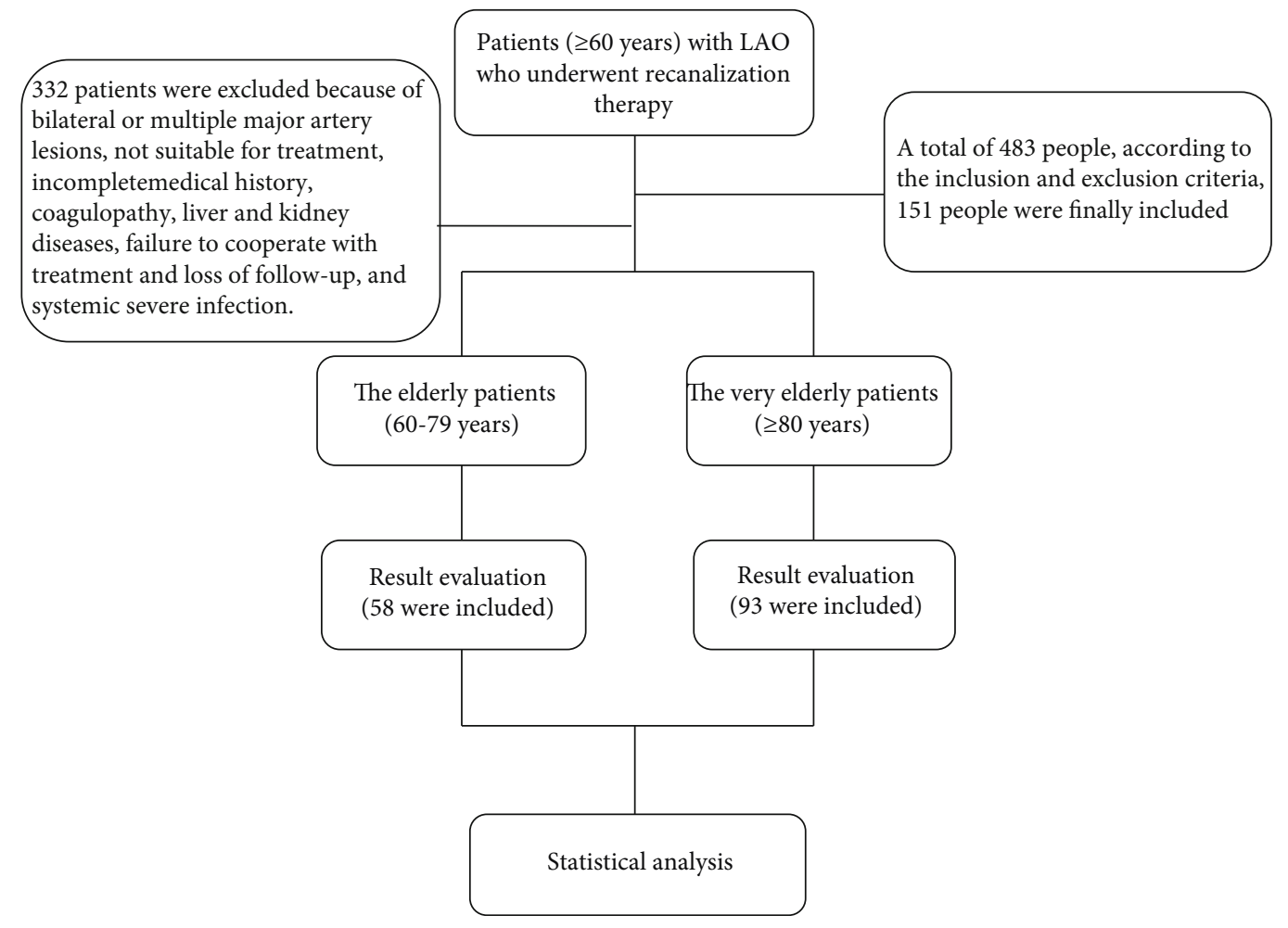

FIgURE 1: Research flow chart.

hemorrhage or cerebral parenchymal hemorrhage with neurological deficit.

2.4. Statistical Analysis. SPSS 22.0 statistical software was used for data analysis. Quantitative data are expressed as mean \pm standard deviation or median (interquartile range (IQR)) depending on whether the data were normally distributed. The $t$-test or the rank-sum test was used to identify differences between two individual groups. Count data are expressed as the number of cases or as a percentage and were compared using the chi-square test. A repeated-measures analysis of variance was used to compare NIHSS and mRS scores at different time points in each group. A $P$ value of $<0.05$ was considered statistically significant. Variables with $P$ values of $<0.1$ in a univariate analysis were included in a multivariate regression analysis. Confounding factors were controlled by stratified analysis and propensity score matching.

\section{Results}

3.1. Patients' Baseline Characteristics. A total of 483 patients were enrolled from November 2013 to July 2017, according to the inclusion and exclusion criteria; 151 people were finally included, all of whom were diagnosed with AIS caused by LAO (Figure 1). There were 74 men (49\% [74/151]) and 77 women (51\% [77/151]). Among them, 38.4\% of patients $(58 / 151)$ belonged to the elderly group (60-79 years of age), and $61.6 \%$ of patients $(93 / 151)$ belonged to the very elderly group (80-93 years of age). There were 18 male patients in the elderly group $(13.03 \%$ [18/58]) and 56 in the very elderly group $(60.22 \%$ [56/93]). The proportion of male patients in the very elderly group was significantly higher compared with that in the elderly group $(P<0.01)$.

Before treatment, there were eight patients $(13.79 \%$ [8/58]) with atrial fibrillation in the elderly group and 27 patients $(29.03 \%$ [27/93]) with atrial fibrillation in the very elderly group $(P=0.875)$.

The incidence of vascular lesions in the elderly and very elderly groups, respectively, was as follows: ICA $(22.4 \%$ vs. $26.9 \%)$, middle cerebral artery $(36.2 \%$ vs. $33.3 \%)$, BA $(29.3 \%$ vs. $29 \%$ ), anterior circulation ( $40.9 \%$ vs. $59.1 \%$ ), and posterior circulation (34.9\% vs. $65.1 \%)$. No significant differences were observed between the two groups $(P>0.05$; Table 1$)$. There was no significant difference in the baseline NIHSS score $(P=0.163)$ or the baseline mRS score $(P=0.935)$ between the elderly group and the very elderly group (Table 1$)$. The specific infarction sites included the basal ganglia, internal capsule, cerebral lobe, watershed region, brainstem, cerebellum, and thalamus (subject to imaging). No significant difference in the number of patients with infarction in these different regions was observed between the two groups $(P=0.980)$ (Table 1$)$.

\subsection{Treatment Results}

3.2.1. $m R S$ Score. Among all patients in the elderly group, 73 (48.3\%) had a favorable 90 -day outcome (mRS score 0-2 points). Compared with the elderly group, the very elderly group had a lower proportion of patients with a favorable outcome (41.6\% vs. $58.6 \%, P=0.046)$ (Figure 2) (Table 2).

3.2.2. Death and Complications. There were 13 deaths $(8.6 \%$ $[13 / 151])$ in the total cohort, and 22 patients $(14.6 \%$ [22/151]) had SICH. No significant difference in the 
TABLE 1: Comparison of baseline characteristics between $<80$ and $\geq 80$ groups.

\begin{tabular}{|c|c|c|c|c|c|c|c|}
\hline \multicolumn{2}{|l|}{ Clinical data } & All $(n=151)$ & $\begin{array}{l}<80 \text {-years-old } \\
\text { group }(n=58)\end{array}$ & $\begin{array}{l}\geq 80 \text {-years-old } \\
\text { group }(n=93)\end{array}$ & Test used & $x^{2} / t / Z$ & $P$ \\
\hline \multirow{2}{*}{ Gender } & Male $(n(\%))$ & $74(49.01)$ & $18(31.03)$ & $56(60.22)$ & \multirow{2}{*}{ Chi-square test } & \multirow{2}{*}{$12.172^{\mathrm{a}}$} & \multirow{2}{*}{$<0.00$} \\
\hline & Female $(n(\%))$ & $77(50.99)$ & $40(68.97)$ & $37(39.78)$ & & & \\
\hline \multicolumn{2}{|l|}{ BMI } & $24.66 \pm 1.73$ & $24.47 \pm 1.83$ & $24.77 \pm 1.67$ & $t$-test & $-1.036^{\mathrm{b}}$ & 0.302 \\
\hline \multicolumn{2}{|c|}{ Time from onset to groin puncture (hours) } & $6.29 \pm 0.72$ & $6.26 \pm 0.68$ & $6.30 \pm 0.76$ & $t$-test & $-0.349^{\mathrm{b}}$ & 0.727 \\
\hline \multicolumn{2}{|c|}{ Time from onset to recanalization (min) } & $40.43 \pm 2.98$ & $40.24 \pm 3.16$ & $40.55 \pm 2.87$ & $t$-test & $-0.615^{b}$ & 0.539 \\
\hline \multirow{5}{*}{ Baseline medical history } & Hypertension $(n(\%))$ & $59(39.07)$ & $23(39.66)$ & $36(38.71)$ & \multirow{3}{*}{ Chi-square test } & \multirow{3}{*}{$0.013^{\mathrm{a}}$} & \multirow{3}{*}{0.908} \\
\hline & Diabetes $(n(\%))$ & $55(36.42)$ & $21(36.21)$ & $34(36.56)$ & & & \\
\hline & Hyperlipidemia ( $n(\%))$ & $48(31.79)$ & $18(31.03)$ & $30(32.26)$ & & & \\
\hline & Coronary heart disease $(n(\%))$ & $53(38.48)$ & $21(29.97)$ & $32(34.41)$ & Chi-square test & $0.002^{\mathrm{a}}$ & 0.965 \\
\hline & Atrial fibrillation $(n(\%))$ & $35(23.18)$ & $8(13.79)$ & $27(29.03)$ & Chi-square test & $0.025^{\mathrm{a}}$ & 0.875 \\
\hline \multirow{6}{*}{ Vessel involved } & Middle cerebral artery $(n(\%))$ & $43(28.48)$ & $21(36.2)$ & $31(33.3)$ & \multirow{4}{*}{ Chi-square test } & \multirow{4}{*}{$0.418^{\mathrm{a}}$} & \multirow{4}{*}{0.741} \\
\hline & Internal carotid artery $(n(\%))$ & $31(20.53)$ & $13(22.4)$ & $25(26.9)$ & & & \\
\hline & Basilar artery $(n(\%))$ & $35(23.18)$ & $17(29.3)$ & $27(29)$ & & & \\
\hline & Vertebral artery $(n(\%))$ & $13(8.61)$ & $7(12.1)$ & $10(10.8)$ & & & \\
\hline & Anterior cerebral artery & $88(58.3)$ & $36(40.9)$ & $52(59.1)$ & \multirow{2}{*}{ Chi-square test } & \multirow{2}{*}{$0.557^{\mathrm{a}}$} & \multirow{2}{*}{0.456} \\
\hline & Posterior cerebral artery & $63(41.7)$ & $22(34.9)$ & $41(65.1)$ & & & \\
\hline \multirow{7}{*}{ Occlusion site } & Basal ganglia & $33(21.9)$ & $13(24.5)$ & $20(20.4)$ & \multirow{7}{*}{ Chi-square test } & \multirow{7}{*}{1.312} & \multirow{7}{*}{0.980} \\
\hline & Inner capsule & $7(4.6)$ & $2(3.8)$ & $5(5.1)$ & & & \\
\hline & Brain lobe & $26(17.2)$ & $10(18.9)$ & $16(16.3)$ & & & \\
\hline & Watershed & $22(14.6)$ & $7(13.2)$ & $15(15.3)$ & & & \\
\hline & Brain stem & $41(27.2)$ & $13(24.5)$ & $28(28.6)$ & & & \\
\hline & Cerebellum & $10(6.6)$ & $3(5.7)$ & $7(7.1)$ & & & \\
\hline & Thalamus & $12(7.9)$ & $5(9.4)$ & $7(7.1)$ & & & \\
\hline \multicolumn{2}{|c|}{ Baseline NIHSS, median (IQR) } & $9(4,16)$ & $11(6,15.25)$ & $8(3,16)$ & $\begin{array}{l}\text { Wilcoxon rank } \\
\text { sum test }\end{array}$ & $4.659^{\mathrm{a}}$ & 0.163 \\
\hline \multicolumn{2}{|c|}{ Baseline mRS, median (IQR) } & $3(2,4)$ & $3(2,4)$ & $3(2,4)$ & $\begin{array}{l}\text { Wilcoxon rank } \\
\text { sum test }\end{array}$ & $0.425^{\mathrm{a}}$ & 0.935 \\
\hline \multicolumn{2}{|l|}{ Acute treatment (rt-PA) } & & & & Chi-square test & $4.247^{\mathrm{a}}$ & 0.001 \\
\hline \multicolumn{2}{|l|}{$0.6 \mathrm{mg} / \mathrm{kg}$} & $63(56.8)$ & $13(20.6)$ & $50(79.4)$ & & & \\
\hline \multicolumn{2}{|l|}{$0.9 \mathrm{mg} / \mathrm{kg}$} & $48(43.2)$ & $10(20.8)$ & $38(79.2)$ & & & \\
\hline
\end{tabular}

a means chi-square test; $\mathrm{b}$ stands for $t$-test.

incidence of mortality or SICH was found between the elderly and the very elderly group (mortality: $10.3 \%$ vs. $7.5 \%, P=0.548$; SICH: $12.07 \%$ vs. $16.13 \%, P=0.561)$ (Table 2). The differences in other complications, including respiratory failure and lung infection, were not statistically significant between the two groups $(P=0.9)$ (Table 2).

3.2.3. Predictor Analysis. Table 3 shows the results of the univariate and multivariate analyses of prognostic factors at 90 days. With the univariate analysis, the factors that predicted a favorable outcome were the male sex (odds ratio $(\mathrm{OR})=3.243,95 \%$ confidence interval (CI): 1.517-6.93, $P=0.002)$ and low-dose rt-PA (OR $=3.170,95 \% \mathrm{CI}: 1.373-7.320, P=0.007)$. The factors that significantly predicted a poor outcome were sex, atrial fibrillation (OR $=4.656,95 \% \mathrm{CI}: 1.772-12.237, P=0.002)$, and thrombolytic therapy $(\mathrm{OR}=2.561, \mathrm{CI}: 1.014-6.465, P=0.047)$. The multivariate regression analysis of predictive factors indi- cated that the male sex and low-dose rt-PA were predictive factors for a favorable outcome.

3.3. Follow-Up Outcome. The duration of clinical follow-up was 90 days. The proportion of patients with a favorable outcome at 90 days $(48.3 \%$ [73/151]) was significantly higher compared with that before treatment (36.4\% [55/151]) $(P=0.036)$. In the elderly group, the proportion of patients with a favorable outcome at 90 days after treatment was significantly higher compared with that before treatment (58.6\% vs. $31 \%$, respectively; $P=0.015$ ). The rate of a favorable outcomes at 90 days $(25.8 \%)$ was not significantly different compared with the rate before treatment $(24.5 \%)$ in the very elderly group $(P=0.791)$. During the follow-up period, there were five patients $(3.3 \%)$ with recurrent ischemic stroke, including two patients in the elderly group and three patients in the very elderly group $(3.4 \%$ vs. $3.2 \%$, 


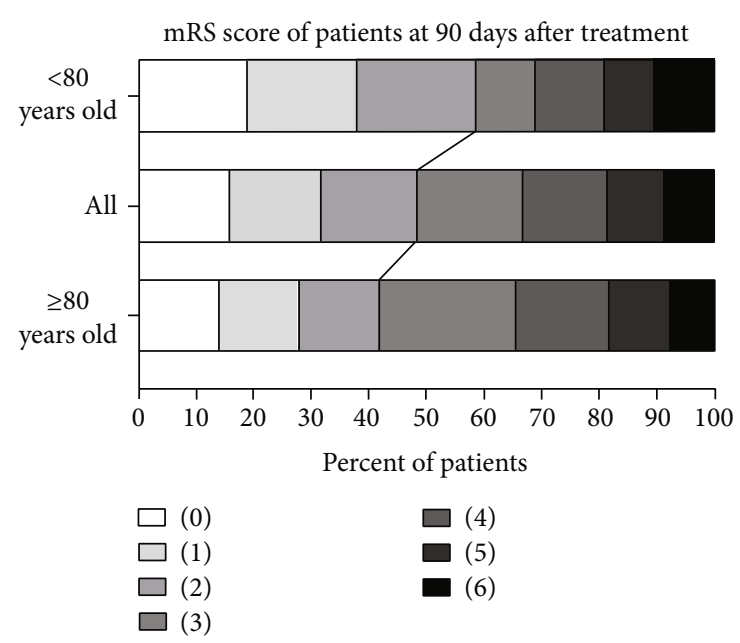

FIGURE 2: Distribution of mRS scores in the elderly group, the very elderly group, and all patients 90 days after treatment. The proportion of patients in each group with different scores was shown (0-6, the higher the score, the more severe the disability). The numbers under the bar represent the proportions of patients in different scoring segments. Due to rounding, the sum of these percentages may not equal 100. The black lines indicate the comparison of the proportion of patients with favorable outcomes (mRS 0-2 points) in different groups. The detailed results were as follows: 90 days after treatment, a total of 73 patients $(48.3 \%)$ had a favorable outcome (mRS $0-2$ ). In the group of $<80$ years old and $\geq 80$ years old, 34 patients $(58.6 \%)$ and 39 patients (41.6\%) had a favorable outcomes, respectively. The ratio of favorable outcomes in the former was higher than that in the latter $(P=0.046)$.

respectively; $P=0.941)$. Four patients $(2.6 \%)$ developed instent restenosis 2 months after surgery, including one patient in the elderly group and three patients in the very elderly group ( $1.7 \%$ vs. $3.2 \%$, respectively; $P=0.576)$.

\section{Discussion}

4.1. Effectiveness Analysis. In this study, the proportion of patients with a favorable outcome 90 days after recanalization treatment differed between the two groups; that is, the proportion of patients with a favorable outcome in the very elderly group was lower compared with that in the elderly group (Figure 2). A meta-analysis involving 1,400 subjects validated this view and proposed that the effectiveness of aortic recanalization therapy in very elderly patients was poorer compared with elderly patients $[10,14]$. Most single-center trials and meta-analyses hold the view that 80 years of age is an independent predictor of functional independence and mortality 30 days after ET, and it is also the demarcation point for a rapid decline in functional prognosis [14-16]. In very elderly patients, functional improvement after treatment is limited by neuronal reserve, remodeling ability, and plasticity [14]. Another possible reason is that difficulty in vascular access and increased vascular distortion or atherosclerotic lesions may affect surgical outcomes $[15,16]$.

Conflicting results have been reported in previous studies. For example, Taichiro et al. showed no significant differ- ence in the favorable outcome rate between the elderly group and the very elderly group ( $42 \%$ vs. $57 \%$, respectively; $P=$ 0.261 ), and the therapeutic effect of ET treatment between the two groups was also comparable [17]. Yasuhiro et al. proposed that age should not be used as a criterion to exclude elderly patients. They believe that improving recanalization techniques could elevate the success rate of treatment in older patients, but there is still no uniform standard on how to improve the technology [18].

In this study, we suggest that in addition to the above reasons, other baseline factors, such as atrial fibrillation and thrombolysis dose, may also be responsible for the poor prognosis of elderly patients. The lower functional independence of patients in the very elderly group before stroke may also reduce treatment effectiveness [19]. Therefore, age may indeed be a factor to consider when deciding whether recanalization therapy should be selected in the clinic.

4.2. Safety Analysis. In this study, we found no significant difference in safety between the two groups within 30 days after treatment. No significant difference was detected in the rate of SICH or mortality between the elderly and very elderly groups $(16.1 \%$ vs. $12.1 \%$, respectively; $P=0.561 ; 7.5 \%$ vs. $10.3 \%$, respectively; $P=0.548$ ). This may be due to the following three reasons. First, a low dose of rt-PA $(0.6 \mathrm{mg} / \mathrm{kg})$ was administered to some patients to improve safety in this study. The conditions suitable for low-dose thrombolysis were as follows: advanced age, mild stroke, relative contraindications, bleeding risk, awakening stroke, and overweight [20]. Twelve patients in the elderly group and 34 patients in the very elderly group underwent low-dose rt-PA treatment. The proportion of patients in the very elderly group who underwent lowdose rt-PA treatment was significantly higher compared with the proportion of patients in the elderly group (36.6\% vs. $20.7 \%$, respectively; $P=0.039$ ). Second, careful treatment selection and active prevention of complications after treatment were adopted to further ensure patient safety. When selecting treatment for patients with a baseline mRS score of $\geq 2$ and an ASPECT score of $\leq 6$, a comprehensive assessment and in-depth communication with family members were carried out to ensure patient safety and successful treatment plan implementation. Finally, the interval from disease onset to treatment was strictly limited to between 4.5 and 6 hours for all patients included in the study [10]. The International Stroke Trial 3 showed no difference in the rate of cerebral hemorrhage and mortality between the very elderly group and the elderly group ( $4 \%$ vs. $3 \%$, respectively; $P=0.667$ ), confirming that thrombolytic therapy is safe in the elderly [21]. The results of the EXTEND-IA and REVASCAT studies have confirmed the safety of IVT and bridge endovascular therapy within 6 hours of AIS onset [22]. Therefore, our results are consistent with previous findings that the safety of recanalization treatment is comparable between the elderly and very elderly groups.

4.3. Influencing Factors. In this study, univariate and multivariate analyses indicated that the male sex and low-dose rt-PA thrombolysis were predictive factors for a favorable outcome. Atrial fibrillation and thrombolytic therapy were 
TABLE 2: Comparison of treatment outcomes between the elderly group and the very elderly group.

\begin{tabular}{|c|c|c|c|c|}
\hline & All & $<80(58)$ & $\geq 80(93)$ & $P$ \\
\hline $\mathrm{mRS}$ at 90 days $(M, \mathrm{IQR})$ & $3(1,4)$ & $2(1,4)$ & $3(1,4)$ & 0.246 \\
\hline $\operatorname{IVT}(M, \mathrm{IQR})$ & $3(1,4)$ & $2(1,4.5)$ & $3(1,4)$ & 0.450 \\
\hline ET $(M, \mathrm{IQR})$ & $3(2,4)$ & $3(1.75,5)$ & $2(0,4)$ & 0.486 \\
\hline $\operatorname{IVT} \& \mathrm{ET}(M, \mathrm{IQR})$ & $2(1,4)$ & $2(0,4)$ & $3(1,4)$ & 0.173 \\
\hline $\begin{array}{l}\text { Before treatment } \\
\text { Favorable outcomes (mRS } 0-2 \text { at } 90 \text { days) }(n, \%)\end{array}$ & $55(36.4)$ & $18(31.0)$ & $37(39.8)$ & 0.277 \\
\hline $\begin{array}{l}90 \text { days after treatment } \\
\text { Favorable outcomes (mRS } 0-2 \text { at } 90 \text { days) }(n, \%)\end{array}$ & $73(48.3)$ & $34(58.6)$ & 39 (41.6) & 0.046 \\
\hline Other outcome (mRS 3-6 at 90 days) $(n, \%)$ & $78(51.7)$ & $24(41.4)$ & $54(58.1)$ & 0.046 \\
\hline $\mathrm{SICH}(n, \%)$ & $22(14.6)$ & $7(12.07)$ & $15(16.13)$ & 0.561 \\
\hline Other complications $(n, \%)$ & $46(30.5)$ & $18(31.0)$ & $28(30.1)$ & 0.904 \\
\hline Death (mRS 6 at 90 days) $(n, \%)$ & $13(8.6)$ & $6(10.3)$ & $7(7.5)$ & 0.548 \\
\hline $\mathrm{mRS}$ at 90 days in different degrees $(n, \%)$ & & & & 0.380 \\
\hline 0 & $24(15.9)$ & $11(19.0)$ & $13(14.0)$ & \\
\hline 1 & $24(15.9)$ & $11(19.0)$ & $13(14.0)$ & \\
\hline 2 & $25(16.6)$ & $12(20.7)$ & $13(14.0)$ & \\
\hline 3 & $28(18.5)$ & $6(10.3)$ & $22(23.7)$ & \\
\hline 4 & $22(14.6)$ & $7(12.1)$ & $15(16.1)$ & \\
\hline 5 & $15(9.9)$ & $5(8.6)$ & $10(10.8)$ & \\
\hline 6 & $13(8.6)$ & $6(10.3)$ & $7(7.5)$ & \\
\hline
\end{tabular}

Normally distributed data is presented as mean \pm standard deviation and compared using independent sample $t$-test. Data that are not normally distributed is expressed as $M$ (P25-P75), and the Wilcoxon rank sum test is used for the comparison between groups. Count data is represented by $n$ (\%), and the comparison between groups is done by the chi-square $\left(\chi^{2}\right)$ test. The multivariate analysis is tested by two-class logistic regression analysis. $P<0.05$ indicates statistical significance.

predictive of a poor outcome. According to previous metaanalyses and multicenter studies, the main factors affecting the effectiveness of recanalization treatment are baseline functional level (a higher baseline NIHSS score), vascular disease location, thrombolysis dose, time from onset to emergency, operation time, and the proportion of patients with mTICI $>2 b / 3[10,14,23]$.

We found no significant difference in the baseline functional level or in the distribution of anterior-circulation and posterior-circulation lesions between the two groups in the present study (Table 1). Moreover, we found that the proportion of male patients with a favorable outcome and a poor outcome was $64.4 \%$ and $34.6 \%$, respectively $(\mathrm{OR}=3.24, P=0.002$ ). Findings from South Korea have shown that the average life expectancy of men is lower than that of women, resulting in a significantly decreased proportion of male patients in the very elderly group, which may have affected the results. Therefore, we speculate that the male sex may be predictive of a favorable outcome. Moreover, an older age and the female sex are also predictors of a poor outcome. Because women have a longer life expectancy than men, women are more likely to be of a higher chronological age when they experience stroke and they may thus have a higher disability rate when classified into the very elderly group $[24,25]$, which is consistent with previous meta-analysis results [14].

The rate of atrial fibrillation in the very elderly group was higher compared with that in the elderly group $(29.03 \%$ vs. $13.79 \%$, respectively; $P=0.031$ ). The proportion of patients with atrial fibrillation in those with favorable and poor out- comes was $11 \%$ and $34.6 \%$, respectively $(\mathrm{OR}=4.656, P=$ 0.02 ), indicating that atrial fibrillation may be an influencing factor of a poor outcome. Similar findings have been reported in previous studies. The incidence of atrial fibrillation confirmed on admission was significantly higher in patients $\geq 80$ years of age compared with patients $<80$ years of age $(69 \%$ vs. $45 \%$, respectively; $P=0.042$ ). Moreover, the incidence of cardiogenic thromboembolism in patients aged $\geq 80$ years was significantly higher compared with those aged $<80$ years ( $94 \%$ vs. 73\%, respectively; $P=0.016$ ). Therefore, we infer that atrial fibrillation may lead to a higher bleeding risk and a poor prognosis. This may be due to large-artery embolism caused by atrial fibrillation, which causes infarction in the area supplied by the middle cerebral artery. Furthermore, atrial fibrillation is often associated with other high-risk factors, such as hypertension and atherosclerosis, which lead to a significantly higher mortality rate compared with patients without atrial fibrillation or patients with tremor [20, 25].

In this study, 111 patients underwent IVT and 40 patients did not undergo IVT, and there was statistical difference between the two $(P<0.05)$. A significant difference was observed between the number of patients with a poor outcome at 90 days and the number of patients with a favorable outcome (68 vs. 43 , respectively; $P<0.001$ ). The multivariate analysis showed that IVT is predictive of a poor outcome $(P=0.047)$, which is contrary to previous studies $[15,16,26,27]$. With a detailed analysis of previous studies, we found that the effect of different rt-PA doses on the results was rarely mentioned. In this study, by 
TABLE 3: Multivariate analysis for predictors of good outcome and poor outcomes.

\begin{tabular}{|c|c|c|c|c|c|}
\hline & \multicolumn{3}{|c|}{ Univariate logistic regressions for outcome } & \multicolumn{2}{|c|}{$\begin{array}{l}\text { Multivariate logistic } \\
\text { regressions for outcome }\end{array}$} \\
\hline & Favorable outcomes $n=73$ & Poor outcomes $n=78$ & $P$ & Odds ratio $(95 \%)$ & $P$ \\
\hline Male & $47(64.4)$ & $27(34.6)$ & $<0.001^{\mathrm{a}}$ & $3.243(1.517,6.93)$ & 0.002 \\
\hline BMI & $24.71 \pm 1.68$ & $24.61 \pm 1.79$ & 0.726 & & \\
\hline Onset to groin time $(\mathrm{h})$ & $6(6,7)$ & $6(6,7)$ & $0.684^{\mathrm{b}}$ & & \\
\hline Onset to IVT time (min) & $40(38,43)$ & $40.5(38,43)$ & $0.419^{c}$ & & \\
\hline Baseline NIHSS & $9(4,15)$ & $9(4,16)$ & $0.761^{\mathrm{c}}$ & & \\
\hline Hypertension & $24(32.9)$ & $35(44.9)$ & $0.131^{\mathrm{a}}$ & & \\
\hline Diabetes mellitus & $31(42.5)$ & $24(30.8)$ & $0.136^{\mathrm{a}}$ & & \\
\hline Hyperlipidemia & $19(26)$ & $29(37.2)$ & $0.141^{\mathrm{a}}$ & & \\
\hline Coronary heart disease & $21(35.1)$ & $32(41)$ & $0.143^{\mathrm{a}}$ & & \\
\hline Arterial fibrillation & $8(11)$ & $27(34.6)$ & $0.001^{\mathrm{a}}$ & $4.656(1.772,12.237)$ & 0.002 \\
\hline Location & & & $0.565^{\mathrm{a}}$ & & \\
\hline Middle cerebral artery $(n(\%))$ & $24(32.9)$ & $28(35.9)$ & & & \\
\hline Internal carotid artery $(n(\%))$ & $21(28.8)$ & $17(21.8)$ & & & \\
\hline Basilar artery $(n(\%))$ & $22(30.1)$ & $22(28.2)$ & & & \\
\hline Vertebral artery $(n(\%))$ & $6(8.2)$ & $11(14.1)$ & & & \\
\hline Treatment options & & & $0.271^{\mathrm{a}}$ & & \\
\hline Age & & & $0.046^{\mathrm{a}}$ & $3.536(1.344,9.306)$ & 0.011 \\
\hline$<80$ & $34(46.6)$ & $24(30.8)$ & & & \\
\hline$\geq 80$ & $39(53.4)$ & $54(69.2)$ & & & \\
\hline rt-PA & & & $<0.001^{\mathrm{a}}$ & $2.561(1.014,6.465)$ & 0.047 \\
\hline No & $30(41.1)$ & $10(12.8)$ & & & \\
\hline Yes & $43(58.9)$ & $68(87.2)$ & & & \\
\hline rt-PA $0.6 \mathrm{mg} / \mathrm{kg}$ & $32(43.8)$ & $14(17.9)$ & $0.001^{\mathrm{a}}$ & $3.170(1.373,7.320)$ & 0.007 \\
\hline
\end{tabular}

"a" indicates chi-square test; "b" indicated $t$-test; "c" indicates $Z$ test. The assignment is as follows: favorable outcomes group $=1$, poor prognosis group $=0$; $0.6 \mathrm{mg} / \mathrm{kg}=1$, and $0.9 \mathrm{mg} / \mathrm{kg}=2$. BMI: body mass index.

comparing the effects of different rt-PA doses, we infer that IVT can affect the outcome of recanalization therapy, but the effect is also influenced by the rt-PA dose. In our study, the number of patients in the elderly and very elderly groups who underwent low-dose rt-PA treatment $(0.6 \mathrm{mg} / \mathrm{kg})$ was significantly different (13 [20.6\%] vs. 50 [79.4\%], respectively; $P=0.001)$. Therefore, we speculate that low-dose rtPA thrombolysis may increase safety but significantly reduce the effectiveness of treatment, thus making IVT a predictive factor for a poor outcome in this study. Similar to our results, Dong et al. recently reported that low-dose rt-PA thrombolysis can reduce the risk of SICH, but it also significantly reduces treatment effectiveness [28].

In previous studies, the influence of age and serum 25 $(\mathrm{OH}) \mathrm{D}$ concentration on the prognosis of stroke has been noted. According to the DNA methylation algorithm, biological age is probably an independent predictor of 3-month mortality after ischemic stroke, which is unrelated to NIHSS score and vascular risk factors [29]. Tu et al. suggest that $25(\mathrm{OH}) \mathrm{D}$ remains an independent prognostic marker of 90-day mortality and functional outcomes in Chinese patients with AIS; lower serum levels of $25(\mathrm{OH}) \mathrm{D}$ are independently associated with the stroke recurrence and mortality at 24 months in ischemic stroke patients $[30,31]$. Cheng et al. suggested that elevated serum hS-CRP and HCY levels were associated with the risk of PSD 1 year after stroke, and this was likely to be a factor influencing the favorable outcomes at 90 days [32]. Further randomized controlled trials are needed to assess the factors that influence the prognosis of stroke in elderly patients.

This study had several limitations. First, this study was a single-center study; thus, the evaluation may not be sufficiently comprehensive. There is a need to further expand the scope of analysis to include other factors, such as ASPECT score, collateral circulation grading, and the relatively small number of cases after grouping. Second, the overall sample size was small, and this limitation was particularly prominent when the three methods were separately counted. This may have led to a decrease in detection efficiency. In future studies, the inclusion criteria should be optimized, the number of subjects should be increased, and data from multiple research centers should be combined for analysis.

\section{Conclusion}

In this study, nearly half of the total cohort achieved a good functional prognosis at 3 months after recanalization. Recanalization had comparable safety between patients aged $\geq 80$ years 
and patients aged 60-79 years. Although there were no significant differences between the two groups in terms of group size, baseline functional score, and vascular lesions, the benefits of recanalization in very elderly patients were not as good as those in elderly patients. Therefore, we infer that age may be an important factor affecting the outcome of aortic recanalization. Furthermore, we identified other influencing factors. Specifically, sex may affect treatment effectiveness, low-dose rt-PA thrombolysis may improve treatment safety, and atrial fibrillation may predict a poor prognosis. In clinical practice, a comprehensive evaluation of certain factors, such as age, must be considered to achieve better treatment outcomes.

\section{Abbreviations}

NIHSS: The National Institutes of Health Stroke Scale

mRS: $\quad$ The modified Rankin scale

DSA: Digital subtraction angiography

AIS: $\quad$ Acute ischemic stroke

LAO: Large artery occlusion

SICH: Symptomatic intracranial hemorrhage

ET: $\quad$ Endovascular thrombectomy

IVT: Intravenous alteplase

rt-PA: Recombinant tissue plasminogen activator

ASPECT: Alberta Stroke Program Early CT

DWI: Diffusion-weighted magnetic resonance imaging

CT: $\quad$ Computed tomography

ICA: Internal carotid artery

VA: Vertebral artery

BA: Basilar artery

M1: $\quad$ Main middle cerebral artery

MRA: Magnetic resonance angiography

mTICI: Modified thrombolysis in cerebral infarction.

\section{Disclosure}

The funders had no role in the design and conduct of the study; in the collection, analysis, and interpretation of the data; and in the preparation, review, or approval of the manuscript.

\section{Conflicts of Interest}

All of the authors have no disclosure-conflict of interest.

\section{Authors' Contributions}

W-JJ contributed to the conception, design, data analysis, and manuscript revision. QW and Y-QZ collected the data and drafted the manuscript. Y-QZ contributed equally to this work and should be considered co-first authors along with Q-W. H-CQ, A-FL, and CL analyzed data and revised the manuscript. Y-DY reviewed and edited the manuscript.

\section{Acknowledgments}

We would like to thank Li Xiang, Department of Record Room, the PLA Rocket Force Characteristic Medical Center, Beijing, 100088, China, for her help in providing data. We thank Emily Woodhouse, $\mathrm{PhD}$, from Liwen Bianji (Edanz) (http://www .liwenbianji.cn/), for editing the English text of a draft of this manuscript. This work was funded by the National Key Basic Research Program of China (973 program) (grant No. 2013CB733800) and National Natural Science Foundation of China (grant Nos. 81471767 and 81871464).

\section{References}

[1] P. B. Gorelick, K. S. Wong, H. J. Bae, and D. K. Pandey, "Large artery intracranial occlusive disease: a large worldwide burden but a relatively neglected frontier," Stroke, vol. 39, no. 8, pp. 2396-2399, 2008.

[2] B.-H. Chao, F. Yan, Y. Hua et al., "Stroke prevention and control system in China: CSPPC-stroke program," International Journal of Stroke, vol. 16, no. 3, pp. 265-272, 2021.

[3] J. S. Balami, B. A. Sutherland, L. D. Edmunds et al., "A systematic review and meta-analysis of randomized controlled trials of endovascular thrombectomy compared with best medical treatment for acute ischemic stroke," International Journal of Stroke, vol. 10, no. 8, pp. 1168-1178, 2015.

[4] O. A. Berkhemer, P. S. Fransen, D. Beumer et al., "A randomized trial of intraarterial treatment for acute ischemic stroke," The New England Journal of Medicine, vol. 372, no. 1, pp. 11-20, 2015.

[5] B. C. Campbell, P. J. Mitchell, T. J. Kleinig et al., "Endovascular therapy for ischemic stroke with perfusion-imaging selection," The New England Journal of Medicine, vol. 372, no. 11, pp. 1009-1018, 2015.

[6] M. Goyal, A. M. Demchuk, B. K. Menon et al., "Randomized assessment of rapid endovascular treatment of ischemic stroke," The New England Journal of Medicine, vol. 372, no. 11, pp. 1019-1030, 2015.

[7] Expert group of scientific statement of Chinese Stroke association, "Scientific statement of Chinese Stroke Association on intravenous thrombolysis in acute ischemic stroke," Chinese Journal of Stroke, vol. 12, no. 3, p. 267, 2017.

[8] E. C. Jauch, J. L. Saver, Adams HP Jr et al., "Guidelines for the early management of patients with acute ischemic stroke: a guideline for healthcare professionals from the American Heart Association/American Stroke Association," Stroke, vol. 44, no. 3, pp. 870-947, 2013.

[9] A. Alawieh, A. Chatterjee, W. Feng et al., "Thrombectomy for acute ischemic stroke in the elderly: a 'real world' experience," Journal of Neurointerventional Surgery, vol. 10, no. 12, pp. 1209-1217, 2018.

[10] M. Goyal, B. K. Menon, W. H. van Zwam et al., "Endovascular thrombectomy after large-vessel ischaemic stroke: a metaanalysis of individual patient data from five randomised trials," Lancet, vol. 387, no. 10029, pp. 1723-1731, 2016.

[11] Chinese stroke association, neurointerventional branch of Chinese stroke society, Chinese association of preventive medicine Interventional group, and committee on prevention and control, "Intravascular therapy for acute ischemic stroke," Chinese Journal Stroke, vol. 13, no. 7, pp. 706-711, 2018.

[12] A. Tamburrini, S. M. Rehman, D. Votano et al., "Penetrating trauma of the thoracic aorta caused by a knitting needle," Annals of Thoracic Surgery, vol. 103, no. 2, article e193, 2017.

[13] X. Li, L. Ling, C. Li, and Q. Ma, "Efficacy and safety of desmoteplase in acute ischemic stroke patients: a systematic review and meta-analysis," Medicine, vol. 96, no. 18, article e6667, 2017. 
[14] A. Alawieh, R. M. Starke, A. R. Chatterjee et al., "Outcomes of endovascular thrombectomy in the elderly: a 'real-world' multicenter study," Journal of Neurointerventional Surgery, pp. 19, 2018.

[15] P. Guedin, A. Larcher, J. P. Decroix et al., "Prior IV thrombolysis facilitates mechanical thrombectomy in acute ischemic stroke," Journal of Stroke and Cerebrovascular Diseases, vol. 24, no. 5, pp. 952-957, 2015.

[16] R. R. Leker, S. Pikis, J. M. Gomori, and J. E. Cohen, "Is Bridging Necessary? A Pilot Study of Bridging versus Primary Stentriever- Based Endovascular Reperfusion in Large Anterior Circulation Strokes," Journal of Stroke and Cerebrovascular Diseases, vol. 24, no. 6, pp. 1163-1167, 2015.

[17] T. Imahori, K. Tanaka, A. Arai, R. Shiomi, D. Fujiwara, and T. Mori, "Mechanical thrombectomy for acute ischemic stroke patients aged 80 years or older Mechanical thrombectomy for acute ischemic stroke in elderly," Journal of Stroke and Cerebrovascular Deseases, vol. 26, no. 12, pp. 2793-2799, 2017.

[18] Y. Kawabata, N. Nakajima, H. Miyake, S. Fukuda, and T. Tsukahara, "Endovascular treatment of acute ischaemic stroke in octogenarians and nonagenarians compared with younger patients," The Neuroradiology Journal, vol. 32, no. 4, pp. 303-308, 2019.

[19] J. F. Kleine, T. Boeckh-Behrens, S. Prothmann, C. Zimmer, and T. Liebig, "Discrepancy between early neurological course and mid-term outcome in older stroke patients after mechanical thrombectomy," Journal of Neurointerventional Surgery, vol. 8, no. 7, pp. 671-676, 2016.

[20] J. L. Saver, M. Goyal, A. Bonafe et al., "Stent-Retriever thrombectomy after intravenous t-PA vs. t-PA alone in stroke," The New England Journal of Medicine, vol. 372, no. 24, pp. 22852295, 2015.

[21] Y. Chen, C.-H. Li, Y.-X. Wang et al., "Safety and effectiveness of intravenous thrombolysis with recombinant tissue plasminogen activator in eighty years and older acute ischemic stroke patients," European Review for Medical and Pharmacological Sciences, vol. 19, no. 10, pp. 1852-1858, 2015.

[22] Z. Di, Z. Shu-ting, and W. Bo, "Interpretation of "Chinese guidelines for diagnosis and treatment of acute ischemic stroke 2018"," Chinese Journal of Contemporary Neurology and Neurosurgery, vol. 19, no. 11, p. 899, 2019.

[23] J. Z. Willey, G. Ortega, P. M. Pego et al., "Thrombolysis in patients aged over 80 years is equally effective and safe, Thrombolysis in patients over 80 years is effective and safe," Journal of Stroke and Cerebrovascular Diseases, vol. 25, no. 6, pp. 1532-1538, 2016.

[24] M. Kelly-Hayes, A. Beiser, C. S. Kase, A. Scaramucci, R. B. D'Agostino, and P. A. Wolf, "The influence of gender and age on disability following ischemic stroke: the Framingham study," Journal of Stroke and Cerebrovascular Diseases, vol. 12, no. 3, pp. 119-126, 2003.

[25] W. Sun, G. Li, X. Zeng et al., "Clinical and imaging characteristics of cerebral infarction in patients with nonvalvular atrial fibrillation combined with cerebral artery stenosis," Journal of Atherosclerosis and Thrombosis, vol. 25, no. 8, pp. 720$732,2018$.

[26] E. A. Mistry, A. M. Mistry, M. O. Nakawah et al., "Mechanical thrombectomy outcomes with and without intravenous thrombolysis in stroke patients: a meta-analysis," Stroke, vol. 48, no. 9, pp. 2450-2456, 2017.

[27] D. Behme, C. Kabbasch, A. Kowoll et al., "Intravenous Thrombolysis Facilitates Successful Recanalization with Stent-
Retriever Mechanical Thrombectomy in Middle Cerebral Artery Occlusions," Journal of Stroke and Cerebrovascular Diseases, vol. 25, no. 4, pp. 954-959, 2016.

[28] Y. Dong, Y. Han, H. Shen et al., "Who may benefit from lower dosages of intravenous tissue plasminogen activator? Results from a cluster data analysis," Stroke \& Vascular Neurology, vol. 5, no. 4, pp. 348-352, 2020.

[29] C. Soriano-Tárraga, E. Giralt-Steinhauer, M. Mola-Caminal et al., "Biological age is a predictor of mortality in ischemic stroke," Scientific Reports, vol. 8, no. 1, p. 4148, 2018.

[30] W. J. Tu, S. J. Zhao, D. J. Xu, and H. Chen, "serum 25hydroxyvitamin D predicts the short-term outcomes of Chinese patients with acute ischaemic stroke," Clinical Science, vol. 126, no. 5, pp. 339-346, 2014.

[31] H. Qiu, M. Wang, D. Mi, J. Zhao, W. Tu, and Q. Liu, "Vitamin $\mathrm{D}$ status and the risk of recurrent stroke and mortality in ischemic stroke patients: data from a 24-month follow-up study in China," The Journal of Nutrition, Health \& Aging, vol. 21, no. 7, pp. 766-771, 2017.

[32] L. S. Cheng, W. J. Tu, Y. Shen, L. J. Zhang, and K. Ji, "Combination of high-sensitivity C-reactive protein and homocysteine predicts the post-stroke depression in patients with ischemic stroke," Molecular Neurobiology, vol. 55, no. 4, pp. 29522958, 2018. 